\title{
Computation of mean size is based on perceived size
}

\author{
Hee Yeon Im and Sang Chul Chong \\ Yonsei University, Seoul, Korea
}

\begin{abstract}
The present study investigated whether computation of mean object size was based on perceived or physical size. The Ebbinghaus illusion was used to make the perceived size of a circle different from its physical size. Four Ebbinghaus configurations were presented either simultaneously (Experiment 1) or sequentially (Experiment 2) to each visual field, and participants were instructed to attend only to the central circles of each configuration. Participants' judgments of mean central circle size were influenced by the Ebbinghaus illusion. In addition, the Ebbinghaus illusion influenced the coding of individual size rather than the averaging. These results suggest that perceived rather than physical size was used in computing the mean size.
\end{abstract}

We commonly encounter groups of similar objects in our environment, such as buildings in a city, cars on the highway, or fruits on a tree. Such objects share spatial locations and resemble one another in shape and size. Although objects within such groups are not identical, they have common features that help us to classify them into categories. How does our visual system represent these common features of similar objects? Some empirical evidence suggests that features shared among all objects of a set can be represented by their statistical properties (Chong \& Treisman, 2003, 2005a, 2005b; Haberman \& Whitney, 2007).

The representation of similar objects on the basis of their statistical properties is advantageous over other possible forms of visual processing. First, statistical representation is useful for understanding and predicting events in a world full of uncertainty. As "intuitive statisticians," we estimate the statistical values - such as average and variance - to be accurate, and we use them both to make optimal inferences in the face of uncertainty (Peterson \& Beach, 1967) and to understand the general trend of events (Beach \& Swenson, 1966). Second, statistical descriptors-such as mean, range, or variance - provide additional global information about an ensemble of objects. For example, when we see a group of dots moving in multiple directions, a complete representation of each individual dot is not very useful in assessing the overall direction of the dots. A representation of the statistical mean direction of the moving dots, however, is more useful in perceiving their global direction (Williams \& Sekuler, 1984). Third, the use of a statistical representation-such as mean size - makes our visual system efficient in coping with redundant information. Statistical representations may extract visual information in the form of gist, which enables efficient object recognition in cluttered scenes and rapid visual scene perception (Oliva \& Torralba, 2006). Finally, statistical representation is more robust to the withdrawal of attention than to the representation of individual features. Alvarez and Oliva (2008) showed that even when local features were so poorly represented that they were hardly identified, it was possible to pool estimates of those local details and to attain an accurate representation of the group, outside the focus of attention.

In size perception, the ability to compute the mean size is surprisingly accurate. Ariely (2001) showed that humans were able to judge the mean size of a set of circles more accurately than they were the size of a single circle selected randomly from a set. Chong and Treisman (2003) extended these findings by demonstrating that the accuracy of mean size judgment remained largely unaffected by exposure duration or delays after exposure. They also found that participants calculated the mean size on a psychological scale. Participants' estimates of the mean size reflected neither the geometric nor the arithmetic mean of the diameters or areas. Instead, the estimates represented the mean circle areas related to a power function with an exponent of 0.76, which is consistent with Teghtsoonian's (1965) findings that the perception of individual circle size is based on a power function with an exponent of 0.76 . When computing the mean size of an ensemble, we most likely use the same psychophysically scaled values, since those are established for an individual size coding. It seems, therefore, that computing the mean size may be based on the perceptual values, which are subject to psychophysical factors. However, there is no direct evidence to support this suggestion. In the present article, we used the Ebbinghaus illusion to test whether computation of mean size was based on perceived size. The Ebbinghaus configuration consists of two identical circles (the targets) placed close to each other, with one surrounded by large 
and the other by small circles (the inducers) (Ebbinghaus, 1902). The contrasting sizes of the two sets of inducers leads to the illusion that the two central circles are different in size, with the central circle surrounded by large inducers appearing smaller than the central circle surrounded by small inducers (Massaro \& Anderson, 1971). Not only does the size contrast modulate the amount of the illusion, the completeness of the surround and the distance between the central circle and inducers (Roberts, Harris, \& Yates, 2005) also affect its magnitude. Given that the perceived size of a target circle of fixed physical size can be changed by adjusting the above factors (size contrast, completeness of inducers, and the distance), we were able to test our hypothesis by manipulating the perceived sizes of elements, leaving the physical sizes of the target circles unaffected. We first measured the magnitudes and the directions (overestimation or underestimation of target circles) of the Ebbinghaus illusion under various configurations. We then tested whether or not the computation of the mean size followed the directions of the effect of the Ebbinghaus illusion. If computation of the mean size is based on perceived sizes of elements, then mean size judgments are expected to be affected by the Ebbinghaus illusion, leading either to overestimation or to underestimation of the mean size of elements, depending on the contexts; otherwise, mean size judgments will be unaffected by the Ebbinghaus illusion as long as the physical sizes of elements are not changed.

\section{EXPERIMENT 1}

We first measured the point of subjective equality (PSE) to evaluate the effect of the Ebbinghaus illusion using a staircase method. The Ebbinghaus illusion was considered to be effective when the measured PSE significantly differed from the presented size of the central circle before the staircase measurement. We then measured the PSE for mean size using the same staircase procedure to test whether participants' judgments of mean size were also influenced by the effect of the Ebbinghaus illusion. If computation of mean size is based on perceived rather than on physical target size, then mean size judgment should be influenced only in those cases in which the illusion differentially affected size perception of individual elements in the left versus the right side of the display.

\section{Method}

Participants. Twenty-four Yonsei University students participated for partial course credit (6 participated in measuring PSE in the test condition, 8 in measuring PSE in control conditions, and 10 in the main experiment). All participants had normal or corrected-to-normal vision. None were aware of the purpose of the experiment. Every aspect of this study was carried out in accordance with the regulations of the Departmental Review Committee of Yonsei University.

Apparatus and Stimuli. The stimuli were created using MATLAB (Psychophysics Toolbox; Brainard, 1997) and presented on a linearized Samsung 21-in. monitor driven by a Pentium IV computer. The frame rate of the monitor was $85 \mathrm{~Hz}$. The participants were seated approximately $90 \mathrm{~cm}$ from the screen with their heads fixed on a chin- and forehead rest, and viewed the display binocularly. At this distance, each pixel was approximately $0.015^{\circ}$ of visual angle.

We generated two different types of Ebbinghaus figures. The first had a conventional configuration (regular Ebbinghaus figure). The inducers in these figures were arranged to occupy $75 \%$ of an imaginary circumference to keep the relative completeness of the surrounding inducers constant (Roberts et al., 2005). Consequently, depending on target size, the number of inducers in each figure was varied from 4 to 12. For the second type (irregular Ebbinghaus figure), jitter was added to randomize the location of the inducers while keeping the average distance between target and each inducer constant. We expected a reduction in magnitude of the illusion due to reduced completeness in the irregular configuration as compared with the regular configuration (Roberts et al., 2005). In this way, we were able to assess whether changes in magnitude of the induced illusion corresponded to changes in mean size judgment. As in the regular Ebbinghaus figures, the number of inducers varied from 4 to 12 .

Each display was divided vertically into two halves, with each containing either one or four Ebbinghaus figures. Each half contained an imaginary $2 \times 2$ matrix in which each cell measured $4.90^{\circ} \times 4.90^{\circ}$. The central position of each figure within each cell was varied randomly within a range of $\pm 0.17^{\circ}$. The diameters of the targets were $0.99^{\circ}, 1.15^{\circ}, 1.30^{\circ}$, and $1.45^{\circ}$, with a mean of $1.22^{\circ}$. The diameter of the inducers was fixed at $1.52^{\circ}$ when the target was smaller than the inducers, and $0.91^{\circ}$ when the target was larger. The distance between the proximal edges of the target and inducers in the regular Ebbinghaus figures was fixed at $0.23^{\circ}$. In order to keep the average distance between target and inducers constant, this distance was either decreased or increased for the irregular Ebbinghaus figures, ranging from $0.17^{\circ}$ to $0.29^{\circ}$.

All stimuli were presented on a light-gray background with a luminance of $18.05 \mathrm{~cd} / \mathrm{m}^{2}$. We used two different colors for targets and inducers (green and red) to ensure that they were easily discriminable. The luminance of the green color was $19.23 \mathrm{~cd} / \mathrm{m}^{2}$, and that of the red was $23.92 \mathrm{~cd} / \mathrm{m}^{2}$. The luminance of red was higher than that of green because a pilot study showed lower visibility for red than for green targets when both were displayed with the same luminance. For half of the participants, the targets were red and the inducers were green; the opposite color scheme was adopted for the other half of the participants.

Design and Procedure. The effectiveness of the configurations to induce the Ebbinghaus illusion was assessed by measuring the PSE prior to the main experiment. A single Ebbinghaus figure was presented to each side of the visual field. Whereas the target size was initially equal, the inducers were larger than the target on one side and smaller than the target on the other. Participants were instructed to press one of two buttons corresponding to the side (left or right) that contained the larger target. A staircase procedure was employed so that one of the target circles was either increased or decreased linearly (by $0.03^{\circ}$ ), depending on the response of the participant. The staircase was terminated after 17 reversals, and the average of the last 4 reversals was defined as PSE. In this manner, the PSEs for Ebbinghaus figures from all experimental conditions were measured. In addition, the same staircase procedure was used to determine the PSE for mean size. In this case, four Ebbinghaus figures were presented to each side of the visual field, and participants were instructed to press one of two buttons corresponding to the side at which they perceived the mean target size to be larger. Again, the target sizes of the four figures in one half of the visual field were increased or decreased (by $0.03^{\circ}$ ) accordingly.

The main experiment included one test condition and four control conditions. In the test condition $\left(\mathrm{R}_{\text {large }} \mathrm{vs}\right.$. $\mathrm{R}_{\text {small }}$ condition), four regular Ebbinghaus figures were presented to each visual field. In one field, the targets were surrounded by large inducers $\left(\mathrm{R}_{\text {large }}\right.$ condition), whereas in the other field, they were surrounded by small inducers $\left(\mathrm{R}_{\text {small }}\right.$ condition). In the first control condition $\left(\mathrm{R}_{\text {large }} \mathrm{vs}\right.$. $\mathrm{I}_{\text {large }}$ condition), only the larger inducers were used, and one of the visual fields contained the irregular Ebbinghaus figures. The second 
A
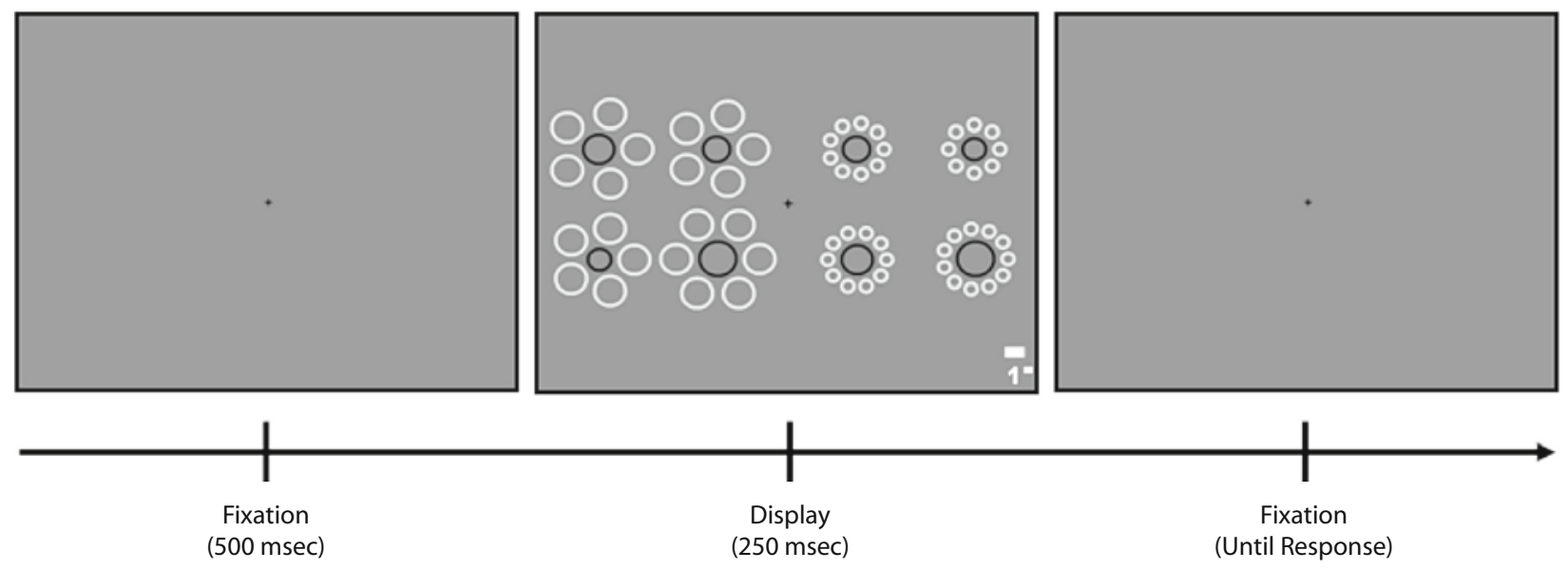

B

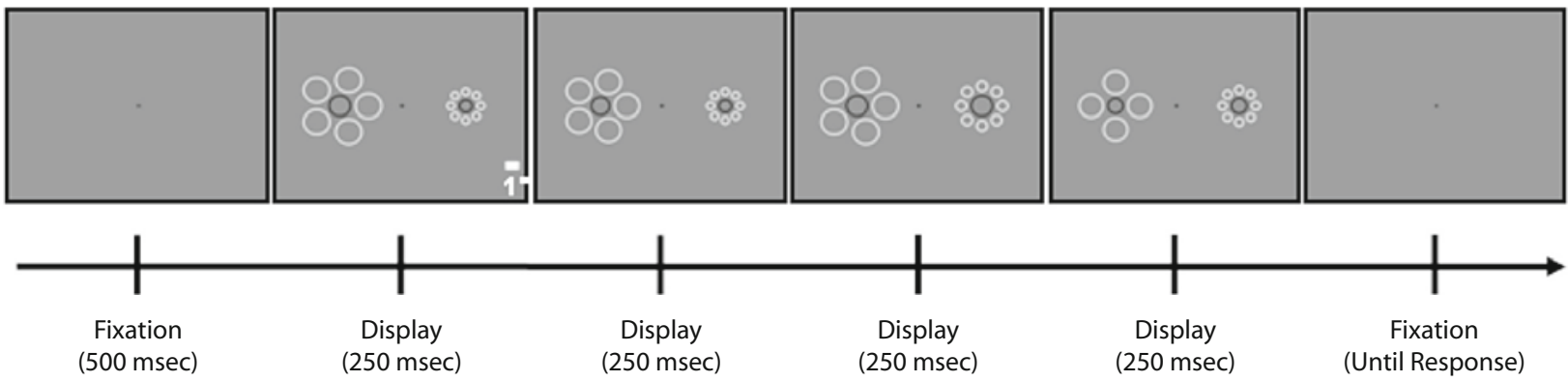

Figure 1. Examples of the procedure in Experiment 1 (A) and in Experiment 2 (B). The Ebbinghaus condition is shown where one visual field has larger inducers, and the other one has smaller inducers. The black circles indicate the targets, and the white circles indicate the inducers.

control condition ( $\mathrm{R}_{\text {small }}$ vs. $\mathrm{I}_{\text {small }}$ condition) was the same as the first except that only the smaller inducers were used. The third and fourth control conditions ( $\mathrm{I}_{\text {large }}$ vs. $\mathrm{I}_{\text {large }}$ and $\mathrm{I}_{\text {small }}$ vs. $\mathrm{I}_{\text {small }}$ conditions) were the same as the first and the second, respectively, except that we used only the irregular configurations in these conditions.

The procedure of Experiment 1 is illustrated in Figure 1A. In all conditions, each trial began with a fixation cross presented for $500 \mathrm{msec}$. The display was then presented for $250 \mathrm{msec}$, followed by a blank screen with a fixation cross that remained until the participant responded. Participants were asked to press one of two buttons corresponding to the side with the larger mean target size. The intertrial interval was $1,000 \mathrm{msec}$. Additionally, participants were instructed beforehand to look at the display as a whole while fixating on the cross in the middle of the display, and to estimate the mean size only for the targets while ignoring the surrounding inducers. Feedback was not provided.

\section{Results and Discussion}

The PSEs were analyzed to test how the Ebbinghaus illusion affected the perceived sizes of targets. On average, it took about 29 trials for participants to achieve 17 reversals. Figure $2 \mathrm{~A}$ shows the size of the illusion as a percentage difference between PSE and the physical size of the target for each condition. The percentage difference between PSE and physical size in the $\mathrm{R}_{\text {large }}$ versus $\mathrm{R}_{\text {small }}$ condition was about $22.31 \%$, which was significantly different from $0[t(5)=4.10, p<.01]$, suggesting that the modulation of the illusion by inducers with different sizes was effective in making perceived sizes of targets different across the visual field in this condition. In contrast, the differences obtained in the control conditions did not significantly differ from $0\left[\mathrm{R}_{\text {large }} \mathrm{Vs} . \mathrm{I}_{\text {large }}(3.65 \%), t(7)=\right.$ $1.62, p=.15 ; \mathrm{R}_{\text {small }}$ vs. $\mathrm{I}_{\text {small }}(2.60 \%), t(7)=1.22, p=$ $.26 ; \mathrm{I}_{\text {large }}$ vs. $\mathrm{I}_{\text {large }}(-2.40 \%), t(7)=1.94, p=.09 ; \mathrm{I}_{\text {small }}$ vs. $\left.\mathrm{I}_{\text {small }}(2.40 \%), t(7)=1.69, p=.14\right]$. This result suggests that in these conditions, the Ebbinghaus illusion did not differentially affect perceived sizes for the left versus right side of the display.

If computation of mean size is based on perceived rather than physical target size, then mean size judgment should be influenced only in those cases in which the Ebbinghaus illusion does have differential effects on perceived sizes of targets for the left versus the right side of the display. Given that only the PSEs of the $\mathrm{R}_{\text {large }}$ versus $\mathrm{R}_{\text {small }}$ condition differed significantly in right versus left visual field, participants' judgments of mean size should be influenced only in the $\mathrm{R}_{\text {large }}$ versus $\mathrm{R}_{\text {small }}$ condition. Figure $2 \mathrm{~B}$ shows the proportion of "larger" responses for the five conditions. The proportion of "larger" responses represents the proportion of trials in which participants judged the mean size of the "PSE-measured side" (the side at which target size was altered according to the staircase procedure) 

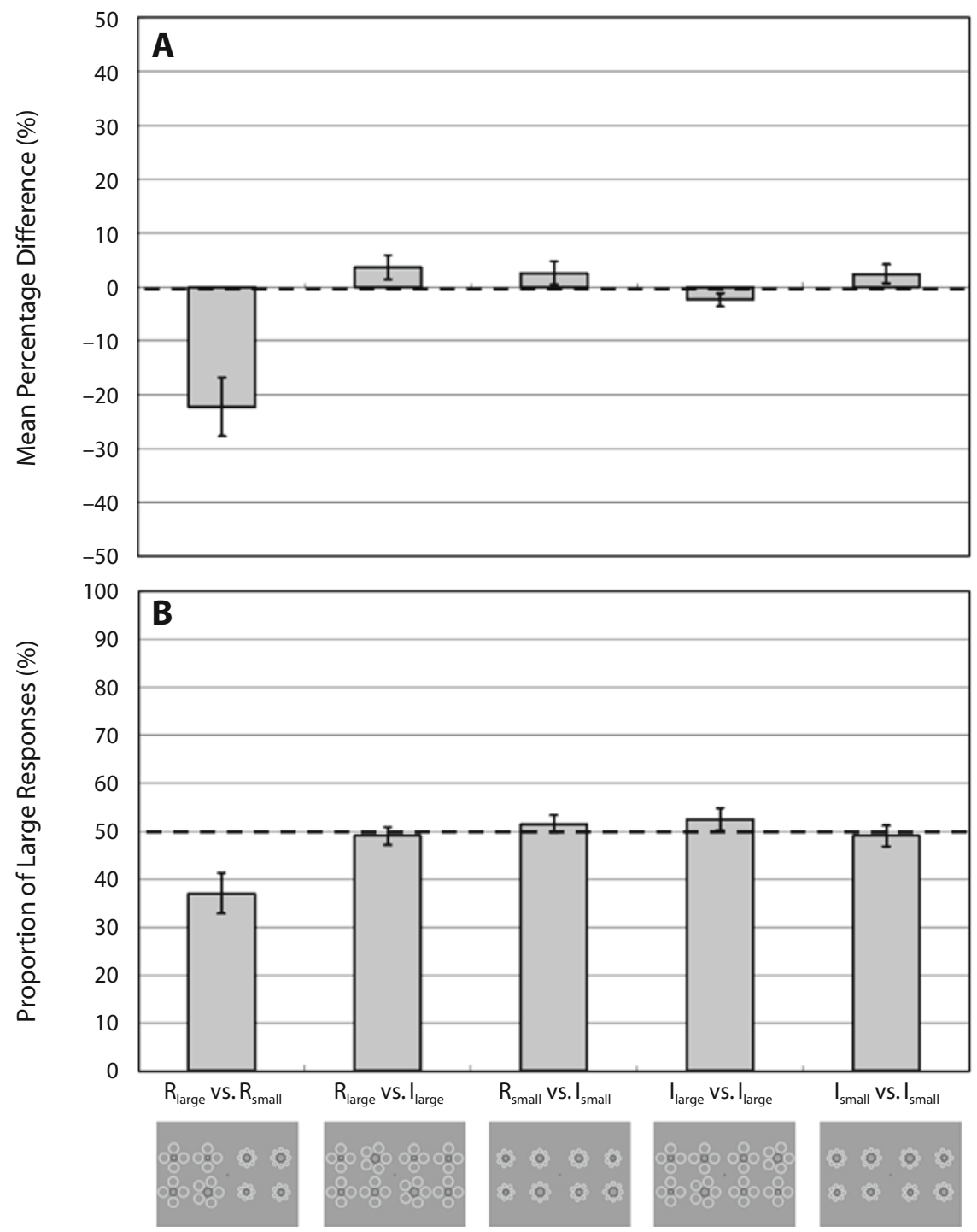

Figure 2. Results of Experiment 1. (A) Mean percentage difference. The dotted line indicates the physical size of targets as a standard. The error bars indicate standard errors. (B) Proportion of "larger" responses for the "PSE-measured side" (the side at which target size was altered according to the staircase procedure) in all conditions. The dotted line indicates chance level, at which the participants' judgments were not biased to any particular visual field. The error bars indicate standard errors.

to be larger. Only the proportion of "larger" responses in the $\mathrm{R}_{\text {large }}$ versus $\mathrm{R}_{\text {small }}$ condition ( $36 \%$ ) was significantly less than $50 \%[t(9)=3.196, p<.05]$, consistent with the PSE results. The PSE of targets with larger inducers was smaller than the presented size. Accordingly, participants perceived targets with larger inducers to have a smaller mean size more often than targets with smaller inducers. These results suggest that the participants' judgments of mean size were indeed influenced by the Ebbinghaus illusion and were therefore made on the basis of perceived rather than physical target size. The proportion of "larger" responses in control conditions did not differ significantly from the chance level, $50 \%\left[\mathrm{R}_{\text {large }}\right.$ vs. $\mathrm{I}_{\text {large }}(49 \%), t(9)=$ $0.44, p=.67 ; \mathrm{R}_{\text {small }}$ vs. $\mathrm{I}_{\text {small }}(52 \%), t(9)=0.93, p=.38$;
$\mathrm{I}_{\text {large }}$ vs. $\mathrm{I}_{\text {large }}(53 \%), t(9)=1.15, p=.28 ; \mathrm{I}_{\text {small }}$ vs. $\mathrm{I}_{\text {small }}$ $(49 \%), t(9)=0.33, p=.75]$; again, this finding is consistent with the PSE results.

\section{EXPERIMENT 2}

In Experiment 2, we investigated whether participants were able to compute the mean size of sequentially presented, temporally intermixed items, as they did over simultaneously presented items in Experiment 1. In $\mathrm{R}_{\text {large }}$ versus $I_{\text {large }}$ and $R_{\text {small }}$ versus $I_{\text {small }}$ conditions of Experiment 1 , we did not find different effects of regular and irregular Ebbinghaus configurations on perceived sizes of targets. Given that the completeness of the surround is an impor- 
tant factor in the Ebbinghaus illusion (Roberts et al., 2005), this null result may have occurred because the irregularity introduced in Experiment 1 was not great enough to sufficiently disturb the completeness of the inducers, as we had intended. Since in Experiment 2 we presented only one Ebbinghaus configuration in each visual field at a time, we were able to make the distance between the proximal edges of the target and inducers in the irregular figures greater than that in Experiment 1, so that inducers in irregular figures had much less completeness than did those in Experiment 1.

\section{Method}

Participants. In Experiment 2, the 2 authors and 27 students participated ( 8 took part in measuring the PSE of the test condition, the mean size, and the target-only condition; 9 took part in measuring the PSE of the other control conditions, and 10 participated in the main experiment). All participants had normal or corrected-to-normal vision. Only the authors were aware of the purpose of the experiment. Every aspect of this study was carried out in accordance with the regulations of the Departmental Review Committee of Yonsei University.

Apparatus and Stimuli. All aspects were identical to those of Experiment 2, except for the following minor changes. The diameters of the targets were $1.07^{\circ}, 1.30^{\circ}, 1.53^{\circ}$, and $1.76^{\circ}$, with a mean of $1.42^{\circ}$. For inducers, eight different diameters were used (large, $1.91^{\circ}, 1.99^{\circ}$, $2.06^{\circ}$, and $2.14^{\circ}, M=2.03^{\circ}$; small, $0.69^{\circ}, 0.76^{\circ}, 0.84^{\circ}$, and $0.92^{\circ}, M=$ $0.80^{\circ}$ ), with all inducers being of the same size for any single trial. In each visual field, only one figure was presented in the center of the matrix at a time, and the distance from the fixation cross was $4.96^{\circ}$. The distance between the proximal edges of the target and inducers in the regular Ebbinghaus figures was fixed at $0.31^{\circ}$. Unlike in Experiment 1 , we increased the distance (ranging from $0.34^{\circ}$ to $1.41^{\circ}$ ) only for the irregular Ebbinghaus figures to ensure that these figures had less completeness than did the regular Ebbinghaus figure.

Design and Procedure. As in Experiment 1, we measured the PSEs for single Ebbinghaus figures and the PSE for mean size of four figures prior to the main experiment. All other aspects were the same as in those Experiment 1, except for those noted below. In measuring the PSE of mean size, four displays-each consisting of two Ebbinghaus figures (one per visual field) - were presented sequentially. Participants were asked to track the target sizes and report, after each trial, which side contained the larger mean size.

For the main experiment, Experiment 2 included two test conditions and five control conditions. In all of these, the display consisted of two Ebbinghaus figures (one in each visual field). The first test condition was the same as in Experiment $1\left(\mathrm{R}_{\text {large }}\right.$ vs. $\mathrm{R}_{\text {small }}$ condition). The second test condition included only irregular figures with smaller inducers in one side and larger inducers in the other side ( $\mathrm{I}_{\text {large }}$ Vs. $\mathrm{I}_{\text {small }}$ condition). The four control conditions were the same as in Experiment 1 ( $\mathrm{R}_{\text {large }}$ vs. $\mathrm{I}_{\text {large }}, \mathrm{R}_{\text {small }}$ Vs. $\mathrm{I}_{\text {small }}, \mathrm{I}_{\text {large }}$ Vs. $\mathrm{I}_{\text {large }}$, and $I_{\text {small }}$ Vs. $I_{\text {small }}$ conditions), but one additional control condition was added in which only the targets were displayed without any inducers (target-only condition).

The procedure of Experiment 2 is illustrated in Figure 1B. In all conditions, each trial began with a fixation cross presented for $500 \mathrm{msec}$, followed by a sequence of four displays (containing two Ebbinghaus figures each, except for the target-only condition, in which only the targets were shown) that were presented for $250 \mathrm{msec}$ each. After the sequence, a blank screen with a fixation cross remained until the participants responded. Participants were asked to compute the mean size of all targets in the sequence and to judge which side of the visual field contained the larger mean size by pressing one out of two buttons at the end of each trial.

\section{Results and Discussion}

As in Experiment 1, PSEs were analyzed for all conditions. For each condition, on average, it took about 34 trials for participants to achieve 17 reversals. Figure $3 \mathrm{~A}$ shows the percentage difference between PSE and physical target size for each condition. In the $\mathrm{R}_{\text {large }}$ versus $\mathrm{R}_{\text {small }}$ condition, there was a significant difference of about $27.77 \%[t(7)=13.64, p<.01]$ between PSE and physical size. A similar result was found for the $\mathrm{I}_{\text {large }}$ versus $\mathrm{I}_{\text {small }}$ condition $[31.47 \% ; t(8)=4.21, p<.01]$, but not for the $I_{\text {large }}$ versus $I_{\text {large }}, I_{\text {small }}$ versus $I_{\text {small }}$, and target-only conditions $\left[\mathrm{I}_{\text {large }}\right.$ vs. $\mathrm{I}_{\text {large }}(0.40 \%), t(8)=0.15, p=.88$; $\mathrm{I}_{\text {small }}$ Vs. $\mathrm{I}_{\text {small }}(4.84 \%), t(8)=1.44, p=.19$; target-only $(1.96 \%), t(7)=1.13, p=.30]$. PSE and physical size was also significantly different in the control $\mathrm{R}_{\text {large }}$ versus $\mathrm{I}_{\text {large }}$ condition [13.89\%; $t(8)=2.58, p<.05]$, and approached significance in the $\mathrm{R}_{\text {small }}$ versus $\mathrm{I}_{\text {small }}$ condition $[15.83 \%$; $t(8)=2.25, p=.055]$. Consequently, the Ebbinghaus illusion differentially influenced the perceived size of the targets in the right versus left visual field in the $R_{\text {large }}$ versus $\mathrm{R}_{\text {small }}$ condition, the $\mathrm{I}_{\text {large }}$ versus $\mathrm{I}_{\text {small }}$ and the $\mathrm{R}_{\text {large }}$ versus $I_{\text {large }}$, and the $R_{\text {small }}$ versus $I_{\text {small }}$ conditions, but not in $I_{\text {large }}$ versus $I_{\text {large }}, I_{\text {small }}$ vs. $I_{\text {small }}$, and target-only conditions.

Figure 3B shows the proportion of "larger" responses for each condition. Again, the percentage of "larger" responses represents the proportion of trials in which the participants judged the mean size of the "PSE-measured" side to be larger. The proportion of "larger" responses (23\%) in the $R_{\text {large }}$ versus $R_{\text {small }}$ condition was significantly lower than $50 \%[t(9)=4.96, p<.01]$, consistent with PSE measurements for this condition. The proportion of "larger" responses $(25 \%)$ in the $\mathrm{I}_{\text {large }}$ versus $\mathrm{I}_{\text {small }}$ condition was also significantly lower than $50 \%[t(9)=8.00, p<.01]$. Participants reported that the mean size of targets with smaller inducers was larger than that of targets with larger inducers, thereby replicating the findings of Experiment 1.

Results for the control conditions revealed that participants did not show any bias for any given condition, consistent with PSE measurements. Specifically, the proportion of "larger" responses for $\mathrm{I}_{\text {large }}$ versus $\mathrm{I}_{\text {large }}, \mathrm{I}_{\text {small }}$ versus $\mathrm{I}_{\text {small }}$, and the target-only conditions did not significantly differ from $50 \%\left[\mathrm{I}_{\text {large }}\right.$ vs. $\mathrm{I}_{\text {large }}(52 \%), t(9)=0.62, p=$ $.55 ; \mathrm{I}_{\text {small }}$ Vs. $\mathrm{I}_{\text {small }}(49 \%), t(9)=0.24, p=.82$; target-only $(54 \%), t(9)=1.20, p=.26]$. Since actual mean sizes for the two visual fields were the same throughout the experiment, a result of $50 \%$ indicates that in these conditions, participants accurately estimated the mean size without any bias; this finding is consistent with the results of previous studies (Ariely, 2001; Chong \& Triesman, 2003). In contrast, the proportion of "larger" responses in $\mathrm{R}_{\text {large }}$ versus $\mathrm{I}_{\text {large }}$ and $\mathrm{R}_{\text {small }}$ versus $\mathrm{I}_{\text {small }}$ conditions differed significantly from $50 \%\left[\mathrm{R}_{\text {large }}\right.$ vs. $\mathrm{I}_{\text {large }}(63 \%), t(9)=3.17, p<$ $.05 ; \mathrm{R}_{\text {small }}$ Vs. $\left.\mathrm{I}_{\text {small }}(61 \%), t(9)=2.30, p<.05\right]$; however, these results were also consistent with the PSE measurements, suggesting that the Ebbinghaus illusion influenced mean size judgments only when the illusion significantly influenced the perceived sizes of targets.

\section{GENERAL DISCUSSION AND CONCLUSIONS}

The present study demonstrates that estimation of mean size can be affected by surrounding the to-be-averaged 

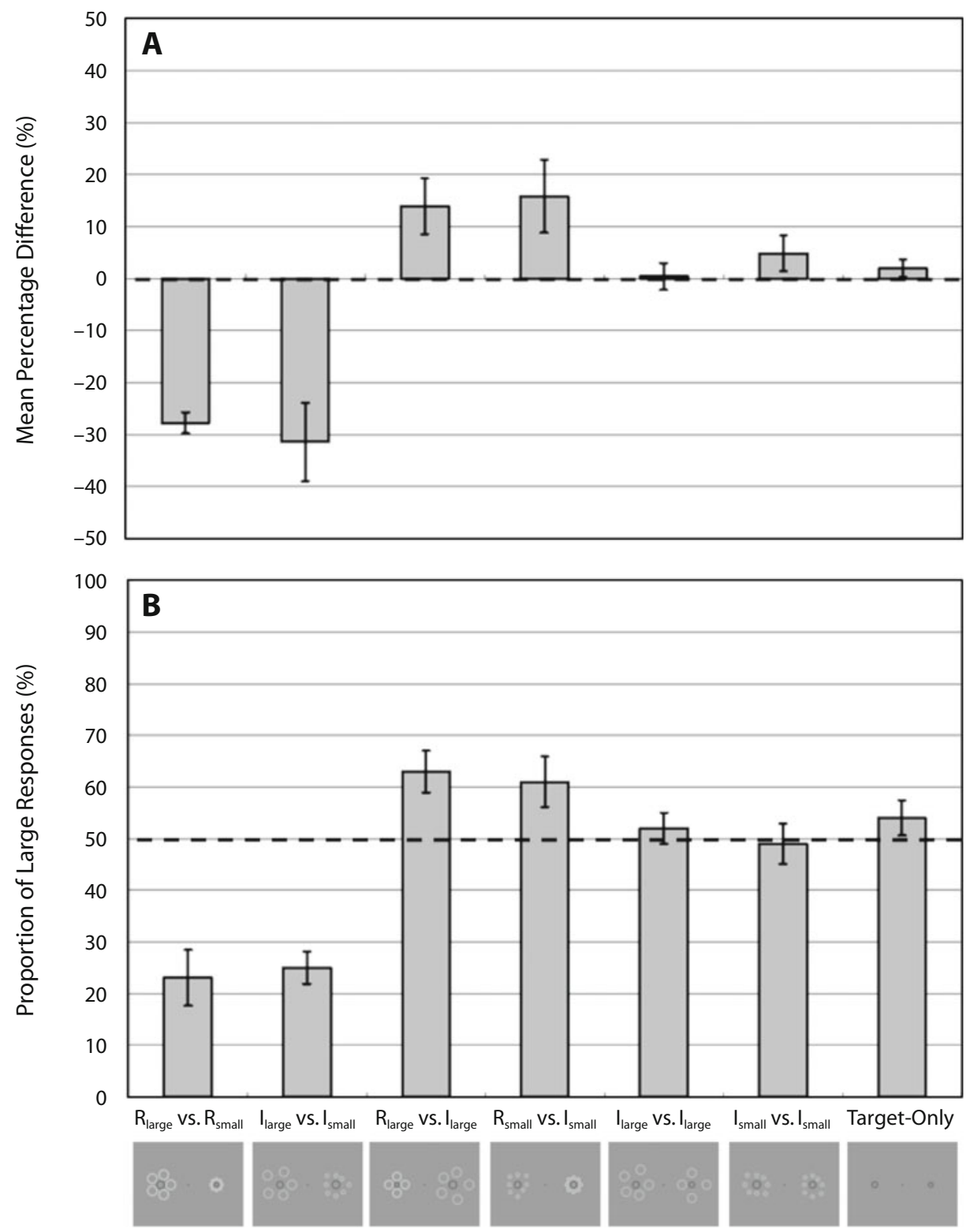

Figure 3. Results of Experiment 2. (A) Mean percentage difference. The dotted line indicates the physical size of targets as a standard. The error bars indicate standard errors. (B) Proportion of "larger" responses for the "PSE-measured side" (the side at which target size was altered according to the staircase procedure) in all conditions. The dotted line indicates chance level, at which the participants' judgments were not biased to any particular visual field. The error bars indicate standard errors.

elements with Ebbinghaus inducers in both parallel (Experiment 1) and serial (Experiment 2) presentations of the targets. We found that the effect of the Ebbinghaus illusion on judgment of mean size was evident when the Ebbinghaus illusion was effective, suggesting that computation of mean size is based on perceived sizes rather than on physical sizes of elements. However, judgment of mean size was not influenced by the Ebbinghaus illusion when there was no effect of the illusion. These results suggest that the participants' judgments of mean size were very accurate and unbiased, given that both the left side and right side actually contained the same mean size, which is consistent with previous studies (Ariely, 2001; Chong $\&$ Treisman, 2003, 2005a, 2005b). Furthermore, the results of Experiment 2 indicate that mean size can be computed from sequentially presented targets, suggesting that the storage of items in memory possibly occurs before mean size computation can occur. These results converge with the findings of Feigenson (2008) that it is possible to perform multiple enumerations over sequences and at distinct locations. In Experiment 2, the individual objects constituting the sets were seen in temporally intermixed 
order so that participants never knew when a certain item, such as the largest one, would be presented in each visual field. This means that all of the items had to be actively maintained and represented throughout the sequence. One might argue that the sequential procedure of Experiment 2 relies on memory and may not be comparable to the procedure in Experiment 1. However, a previous study (Chong \& Treisman, 2003) found little effect of a memory delay of up to $2 \mathrm{sec}$ on mean size computation. Since it took only $1 \mathrm{sec}$ to present all of the figures in Experiment 2, it is unlikely that the memory delay would influence the results in regard to mean size estimation.

The method we used involves comparing the means on the right and left sides of the screen. One may ask whether participants were able to perform the task by focusing attention on samples of just one or two items from each side (Myczek \& Simons, 2008). For example, one plausible sampling strategy would be for participants to simply compare the largest items on each side. In fact, even if participants had picked only the largest target from each visual field and compared them with each other without computing the mean size of the entire array, the same results as those expected would have been obtained if the participants had considered all of the targets. In Experiment 2, however, only one configuration was seen in each visual field at a time, and the sequence of targets was temporally intermixed. Therefore, participants could not simply choose one among four targets, such as the largest one or the smallest one from each visual field. This is consistent with findings of Chong, Joo, Emmanouil, and Treisman (2008), who showed that participants were not liable to use sampling strategies such as using the largest size or foveal items.

Unlike in Alvarez and Oliva (2008), in our task, participants could not rely on location-based selection alone because targets were presented both in the left and in the right visual fields. They could not rely solely on featurebased selection by color, either. Consequently, they had to use a combination of location and color to select the targets. One might have doubts that participants were able to select only the targets to average in the display. In fact, it is possible that selection for targets was not quite perfect and the participants somehow took the inducers into account. However, if this were the case, the results would have been the complete opposite of those found. Although the mean size of all presented circles was always larger in the visual field with larger inducers, participants chose the visual field with smaller inducers in the $R_{\text {large }}$ versus $R_{\text {small }}$ condition as having a larger mean target size. For example, in the $\mathrm{R}_{\text {large }}$ versus $\mathrm{R}_{\text {small }}$ condition, the mean size of the entire display with larger inducers was, on average, $152 \%$ greater than that with smaller inducers. Although the inducers might have been processed to some degree (affecting the individual size coding of the targets), it is less likely that participants used the mean size of the entire display in their calculations without selecting the targets from the display. The participants in our study successfully selected only the targets to average in the display, using both location- and color-based selection

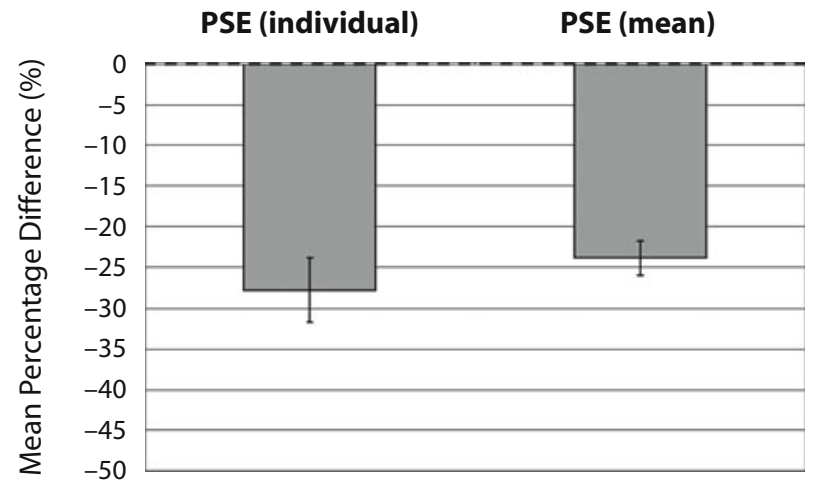

Figure 4. Comparison of PSE of single size and PSE of mean size of the smallest targets used in Experiment 2. The dotted line indicates the physical size of the targets as a standard, and the error bars indicate standard errors.

mechanisms, consistent with previous studies (Serences \& Boynton, 2007; Sohn, Chong, Papathomas, \& Vidnyanszky, 2005).

If the Ebbinghaus illusion indeed influences mean size computation of a group of circles, one important question remains: Is the perceived size of each item first modulated by the Ebbinghaus illusion and does it then participate in the averaging, or are the items first averaged and is the averaged size then modulated by the inducers in the Ebbinghaus configuration? The results of Experiment 2 may provide an answer. In the $\mathrm{R}_{\text {large }}$ versus $\mathrm{R}_{\text {small }}$ condition, as shown in Figure 4, the PSE of the mean size for targets in Ebbinghaus figures was different from their physical mean size by $23.84 \%[t(7)=6.03, p<.01]$, and the PSE for a single target in an Ebbinhaus figure was also different from its physical size, by $27.77 \%[t(7)=13.64$, $p<.01]$. The difference of $23.84 \%$ between PSE of the mean size and actual mean size indicates that the size of targets with smaller inducers would have to be reduced by $23.84 \%$ in order to have the same perceived mean size as targets without inducers; the same logic can be applied to the PSE of individual sizes. The difference between PSEs of the mean size and that between PSEs of the single target did not significantly differ from each other $[t(7)=1.05$, $p=.33$ ], suggesting that the Ebbinghaus illusion had the same effect on individual size coding and mean size estimates. Thus, the Ebbinghaus illusion seems to influence the coding of individual size rather than directly influencing the computation of mean size itself. If individual sizes are first averaged and the averaged size is modulated by the illusion, the Ebbinghaus illusion would not necessarily produce the same amount of effect on individual size coding and mean size estimates; it would affect only mean size estimates, leaving the individual size coding unaffected. Therefore, our results suggest that individual target sizes are more likely influenced by the illusion first, and then participate in averaging, rather than the reverse. To test this directly, we conducted an additional experiment. We used the same stimuli and procedure as in Experiment 1, but varied the configuration of target and inducers in some 


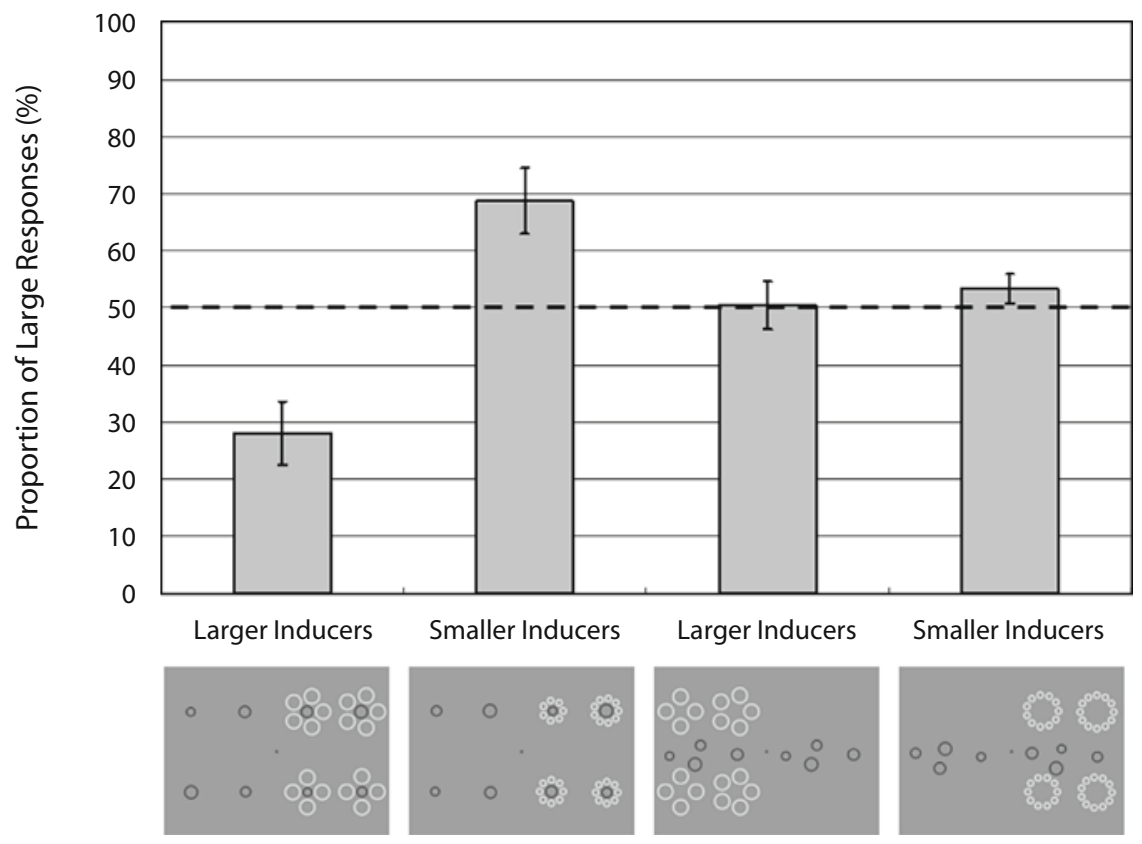

Figure 5. Stimuli and results of the additional Experiment. (A) Trials in which inducers were closely surrounding targets. (B) Trials in which inducers were not surrounding targets but sharing the general region. The dotted line indicates chance level, at which the participants' judgments were not biased to any particular visual field. The error bars indicate standard errors.

trials. If the Ebbinghaus illusion affects the computation of mean size itself rather than individual size coding, the inducers would be equally effective as long as they were in the same general region, even when they were not closely surrounding the individual targets. However, if the illusion affects individual size coding, followed by averaging, the inducers would have to be around the individual targets in order to be effective. Figure 5 shows a sample display and results of the additional experiment. We found that the illusion was effective only when the inducers were closely surrounding the targets [larger inducers, $t(5)=3.952$, $p<.05$; smaller inducers, $t(5)=3.232, p<.05$ ], but not when the inducers were simply in the general region of the targets [larger inducers, $t(5)=0.099, p=.925$; smaller inducers, $t(5)=1.305, p=.249$ ]. These results suggest that the Ebbinghaus illusion modulates individual size coding first; then the perceived sizes are used for mean size computation.

Although many previous studies have emphasized a number of important factors influencing the magnitude of the Ebbinghaus illusion, such as size contrast or distance, specific interactions between these factors are still under discussion. According to size-contrast aspect, larger inducers make the target look smaller, and smaller inducers make the target look larger (Coren \& Enns, 1993; Coren \& Miller, 1974; Ehrenstein \& Hamada, 1995; Massaro \& Anderson, 1971; Müller \& Busch, 2006; Roberts et al., 2005). According to distance aspect, however, the perceived size of a target is determined by the distance between the target and its surrounding inducers: The target appears larger when the inducers are close to the target and smaller when the inducers are far from the target (Girgus, Coren, \& Agdern, 1972; Jaeger, 1978; Jaeger \& Grasso, 1993; Roberts et al., 2005; Weintraub \& Schneck, 1986). The results from the $\mathrm{R}_{\text {large }}$ versus $\mathrm{I}_{\text {large }}$ and $\mathrm{R}_{\text {small }}$ versus $I_{\text {small }}$ conditions of Experiment 2 can provide insight into the interaction of these two factors. We found differential effects of regular and irregular Ebbinghaus figures on perceived sizes of targets in the $R_{\text {large }}$ versus $I_{\text {large }}$ and $\mathrm{R}_{\text {small }}$ versus $\mathrm{I}_{\text {small }}$ conditions of Experiment 2; mean size was judged to be larger in the case of regular Ebbinghaus figures than in the case of irregular Ebbinghaus figures, irrespective of size contrast. However, we did not find a difference between perceived sizes of targets for regular and irregular Ebbinghaus figures in $\mathrm{R}_{\text {large }}$ versus $\mathrm{I}_{\text {large }}$ and $R_{\text {small }}$ versus $I_{\text {small }}$ conditions of Experiment 1. Note that inducers and targets were always closer in regular Ebbinghaus figures than in irregular Ebbinghuas figures in Experiment 2, whereas the average distances between target and each inducer in regular and irregular Ebbinghaus figures were constant in Experiment 1. These results suggest that the distance between target and inducers might be a primary factor determining the magnitude of the Ebbinghaus illusion. Moreover, the magnitude of the illusion was not significantly different $[t(8)=0.26, p=$ $.805]$ when the distances between target and inducers in irregular Ebbinghaus figures were the same in $\mathrm{R}_{\text {large }}$ versus $\mathrm{I}_{\text {large }}$ and $\mathrm{R}_{\text {small }}$ versus $\mathrm{I}_{\text {small }}$ conditions. These results suggest that the distance between the target and inducers is a more important factor in determining the magnitude of the Ebbinghaus illusion than the size contrast. When the distance factor was controlled, as in Experiment 1, the 
effect of size contrast remained effective as it was in the $\mathrm{R}_{\text {large }}$ versus $\mathrm{R}_{\text {small }}$ condition of Experiment 1 . However, when the distance between target and inducers was varied, as in Experiment 2, the magnitude of the illusion seemed to depend more on the distance than on size contrast.

Previous studies have suggested that the computation of mean size is probably an early visual process. For example, judgment of mean size was not affected by the number or density of items presented, indicating that mean size is likely to be computed automatically and in parallel (Chong \& Treisman, 2005a). In addition, performing an extra task did not interfere with mean size computation, suggesting that it may be a preattentive process (Chong \& Treisman, 2005b). The Ebbinghaus illusion also seems to occur early in visual processing. Busch and Müller (2004; Müller \& Busch, 2006) found that the detection of a target was independent of the number of distractors in visual search, even when the target and distractors were surrounded by inducers. The absence of an effect of the number of distractors in visual search was considered a hallmark of preattentive processing (Treisman \& Gelade, 1980). In addition, Choplin and Medin (1999) found that the Ebbinghaus illusion was affected by the degree of perceptual similarity between the perimeters of the inducers and the targets, especially if they were distinguished by early features, such as curvilinear circles among rectilinear triangles. However, the conceptual similarity of factors such as the complex shapes of target and inducers did not matter. Their findings suggest that the perceived size of the target circle in the Ebbinghaus figure might be computed early in the visual system. In addition to the psychophysical studies, an fMRI study recently reported that early visual areas, such as V1, can represent the perceived size of an object and reflect the apparent size modulation of the object (Murray, Boyaci, \& Kersten, 2006). Taken together, the perceived size of an individual object can be scaled and represented even in very early visual areas. If this is the case, then where in the processing stream is mean size computed? Is mean size computed before or after modulation of perceived size by the Ebbinghaus illusion? Given that the participants' judgments of mean size were influenced by the apparent size modulation of the Ebbinghaus illusion in the present study, it is possible that mean size is computed after or at least coinciding with the modulation of perceived size.

In conclusion, we showed that computation of mean size is based on perceived size. Only when the Ebbinghaus illusion affected the perception of a target were the participants' judgments of the mean size influenced by the illusion. The Ebbinghaus illusion influenced individual size coding rather than computation of the mean itself.

\section{AUTHOR NOTE}

This work was supported by Korea Research Foundation Grant KRF-2006-332-H00039, funded by the Korean Government (MOEHRD). For helpful comments and discussion on this manuscript, we thank Moritz Stolte. We also thank Liqiang Huang for the suggestion of a new experiment and other reviewers for their helpful comments. Address correspondence to S. C. Chong, Department of Psychology, Yonsei University, Sinchon-dong, Seodaemun-gu, Seoul, Korea (e-mail: scchong@yonsei .ac.kr).

\section{REFERENCES}

Alvarez, G. A., \& Oliva, A. (2008). The representation of simple ensemble visual features outside the focus of attention. Psychological Science, 19, 392-398. doi:10.1111/j.1467-9280.2008.02098.x

ARIELY, D. (2001). Seeing sets: Representation by statistical properties. Psychological Science, 12, 157-162. doi:10.1111/1467-9280.00327

BEACH, L. R., \& SwENSON, R. G. (1966). Intuitive estimation of means. Psychonomic Science, 5, 161-162.

BRAINARD, D. H. (1997). The psychophysics toolbox. Spatial Vision, 10, 433-436. doi:10.1163/156856897X00357

Busch, A., \& MüLler, H. J. (2004). The Ebbinghaus illusion modulates visual search for size-defined targets: Evidence for preattentive processing of apparent object size. Perception \& Psychophysics, 66, 475-495.

Chong, S. C., Joo, S. J., Emmanouil, T. A., \& Treisman, A. (2008). Statistical processing: Not so implausible after all. Perception \& Psychophysics, 70, 1327-1334.

Chong, S. C., \& Treisman, A. (2003). Representation of statistical properties. Vision Research, 43, 393-404. doi:10.1016/S00426989(02)00596-5

Chong, S. C., \& Treisman, A. (2005a). Statistical processing: Computing the average size in perceptual groups. Vision Research, 45, 891-900. doi:10.1016/j.visres.2004.10.004

Chong, S. C., \& Treisman, A. (2005b). Attentional spread in the statistical processing of visual displays. Perception \& Psychophysics, 67, 1-13.

Choplin, J. M., \& Medin, D. L. (1999). Similarity of the perimeters in the Ebbinghaus illusion. Perception \& Psychophysics, 61, 3-12.

Coren, S., \& Enns, J. T. (1993). Size contrast as a function of conceptual similarity between test and inducers. Perception \& Psychophysics, 54, 579-588.

Coren, S., \& Miller, J. (1974). Size contrast as a function of figural similarity. Perception \& Psychophysics, 16, 355-357.

Ebirnghaus, H. (1902). Grundzuge der Psychologie (Vol. 1., Band 2. Theil.). Leipzig: Viet \& Co.

Ehrenstein, W. H., \& Hamada, J. (1995). Structural factors of size contrast in the Ebbinghaus illusion. Japanese Psychological Research, 37, 158-169.

Feigenson, L. (2008). Parallel non-verbal enumeration is constrained by a set-based limit. Cognition, 107, 1-18. doi:10.1016/j cognition.2007.07.006

Girgus, J. S., Coren, S., \& Agdern, M. (1972). The interrelatioship between the Ebbinghaus and the Delboef illusions. Journal of Experimental Psychology, 95, 453-455. doi:10.1037/h0033606

Haberman, J., \& Whitney, D. (2007). Rapid extraction of mean emotion and gender from sets of faces. Current Biology, 17, 751-753.

JAEGER, T. (1978). Ebbinghaus illusions: Size contrast or contour interaction phenomena? Perception \& Psychophysics, 24, 337-342.

JaEger, T., \& Grasso, K. (1993). Contour lightness and separation effects in the Ebbinghaus illusion. Perceptual \& Motor Skills, 76, 255-258.

Massaro, D. W., \& Anderson, N. H. (1971). Judgmental model of the Ebbinghaus illusion. Journal of Experimental Psychology, 89, 147-151. doi:10.1037/h0031158

MÜLleR, H. J., \& BuSCH, A. (2006). Visual search for size-defined target objects is modulated by the Ebbinghaus apparent-size illusion: Facilitatory and inhibitory effects of the context objects. Perception, 35, 671-700. doi:10.1068/p5139

Murray, S. O., Boyaci, H., \& Kersten, D. (2006). The representation of perceived angular size in human primary visual cortex. Nature Neuroscience, 9, 429-434. doi:10.1038/nn1641

MyczeK, K., \& Simons, D. J. (2008). Better than average: Alternatives to statistical summary representations for rapid judgments of average size. Perception \& Psychophysics, 70, 772-788.

Oliva, A., \& Torralba, A. (2006). Building the gist of a scene: The role of global image features in recognition. Progress in Brain Research: Visual Perception, 155, 23-36.

Peterson, C. R., \& Beach, L. R. (1967). Man as an intuitive statistician. Psychological Bulletin, 68, 29-46.

Roberts, B., Harris, M. G., \& YATES, T. A. (2005). The roles of inducer size and distance in the Ebbinghaus illusion (Titchener circles). Perception, 34, 847-856. doi:10.1068/p5273 
Serences, J. T., \& Boynton, G. M. (2007). Feature-based attentional modulations in the absence of direct visual stimulation. Neuron, 19, 301-312.

Sohn, W., Chong, S. C., Papathomas, T. V., \& Vidnyanszky, Z. (2005). Cross-feature spread of global attentional modulation in human area MT+. NeuroReport, 16, 1389-1393. doi:10.1097/01 .wnr.0000174059.57144.62

Teghtsoonian, M. (1965). The judgment of size. American Journal of Psychology, 78, 392-402. doi:10.2307/1420573

Treisman, A., \& Gelade, G. (1980). A feature integration theory of attention. Cognitive Psychology, 12, 97-136. doi:10.1016/00100285(80)90005-5
Weintraub, D. J., \& Schneck, M. K. (1986). Fragments of Delboef and Ebbinghaus illusions: Contour/context explorations of misjudged circle size. Perception \& Psychophysics, 40, 147-158.

Williams, D. W., \& Sekuler, R. (1984). Coherent global motion percepts from stochastic local motions. Vision Research, 24, 55-62. doi:10.1016/0042-6989(84)90144-5

(Manuscript received May 30, 2008;

revision accepted for publication August 28, 2008.) 